\title{
EFFECTIVE STABILITY IN REVERSIBLE SYSTEMS
}

\author{
AMADEU DELSHAMS \\ Departament de Matemàtica Aplicada I, Universitat Politècnica de Catalunya \\ Diagonal 647, E-08071 Barcelona, Spain
}

AND J. TOMÁS LÁZARO

Departament de Matemàtica Aplicada i Telemàtica

Universitat Politècnica de Catalunya

Victor Balaguer s/n, E-08800, Vilanova i la Geltrú, Barcelona, Spain

\section{Introduction}

It is difficult, in the theory of dynamical systems, to draw a boundary line between conservation laws and symmetries because often their effects on the dynamics can be almost the same. An important example can be the case of motions in Hamiltonian and reversible systems. For instance, it is well-known that the Kolmogorov-Arnol'd-Moser theory applies in both type of systems (see $[1,2,12,11]$ ). In the theory of Hamiltonian systems we also have the Nekhoroshev theorem [7], that leads to the concept of effective stability.

In this paper we present a procedure to put in normal form a nearly-integrable reversible system, not necessarily a Hamiltonian system. Furthermore, non-resonant stability estimates are obtained. As an application we discuss the case of $n$ harmonic oscillators with frequencies satisfying Diophantine conditions. We will consider only linear reversibility because it appears often in Hydrodynamics, Thermodynamics, Fluid Mechanics and Electromagnetism.

Given a linear involution $R$ of $\mathbb{R}^{2 n}$, we will say that a dynamical system is $R$-reversible, or simply reversible, if it is invariant under the transformation $X \longmapsto R X, t \longmapsto-t$, where $X \in \mathbb{R}^{2 n}$. In other words, we can say that an autonomous system $\dot{X}=\mathcal{F}(X)$ is reversible if it verifies $\mathcal{F}=-\square \mathcal{F}$, where the functional operator $\square$ is defined as $\square \mathcal{F}=R \cdot \mathcal{F} \circ R$. Note that an important difference between reversible systems and Hamiltonian systems is that we have no energy conservation in the first ones.

\section{Normal Forms via the Lie series method}

We seek for a suitable transformation $\Phi$ that brings our vector field $\mathcal{F}$ into a normal form $\mathcal{F}^{*}=\Phi^{*} \mathcal{F}$ preserving the reversible character. Let us consider autonomous systems of the form $\dot{X}=\mathcal{S}(X)$, with $X \in \mathbb{R}^{2 n}$, and satisfying the condition $\mathcal{S}=\square \mathcal{S}$. Then, the mapping defined as the flow at time $t$ of this kind of systems, $\psi_{t}^{S}$, is a transformation that keeps the reversible character invariant. We will call them reversible transformations and we will work with $\psi^{S}=\psi_{t=1}^{S}$. We construct the successive transformations with the help of the Lie series method for vector fields, as in [4]. A well-known obstruction to the pass to normal form is found on the resonances or near them. Given a module $\mathcal{M} \subset \mathbb{Z}^{n}$ we have the resonant manifold associated to it, $S_{\mathcal{M}}=\{I \in \mathcal{G}: k \cdot \omega(I)=0 \quad \forall k \in \mathcal{M}\}$. We say that a function $g(I, \varphi)$ is in normal form with respect to $\mathcal{M}$ of degree $K$ if its Fourier 
series expansion in the angular variables has the form

$$
g(I, \varphi)=\sum_{k \in \mathcal{M},|k|_{1} \leq K} g_{k}(I) e^{i k \cdot \varphi},
$$

where $|k|_{1}=\sum_{i=1}^{n}\left|k_{i}\right|$, and $k=\left(k_{1}, k_{2}, \ldots, k_{n}\right) \in \mathbb{Z}^{n}$. Then we express it by writing $f \in \boldsymbol{R}(\mathcal{M}, K)$. We say that a vector field $\mathcal{F}(I, \varphi) \in \boldsymbol{R}(\mathcal{M}, K)$ if each one of its components belongs to $\boldsymbol{R}(\mathcal{M}, K)$.

Now, we describe briefly the iterative process that leads our reversible vector field $\mathcal{F}(I, \varphi)$ to normal form. First of all, we restrict our $\mathcal{F}$ to a subset $G \subset \mathcal{G}$ where we can assume the frequency set $\omega(G)$ is non resonant modulo $\mathcal{M}$ up to order $K$. We start with $\mathcal{F}(I, \varphi)=\Omega(I)+\mathcal{Z}(I, \varphi)+\mathcal{R}(I, \varphi)$, where $\Omega(I)=(0, \omega(I))$ and $\mathcal{Z} \in \boldsymbol{R}(\mathcal{M}, K)$. The transformation $\Phi$ leading our vector field to normal form is composed by reversible transformations $\psi^{(1)}, \psi^{(2)}, \ldots$ as described before. At the step $q$, we have then $\mathcal{F}^{(q)}=$ $\Phi^{(q)^{*}} \mathcal{F}=\Omega+\mathcal{Z}^{(q)}+\mathcal{R}^{(q)}$, with $\Phi^{(q)}=\psi^{(1)} \circ \psi^{(2)} \circ \ldots \psi^{(q)}$ and $\Phi^{(q)^{*}} \mathcal{F}=\left(D\left(\Phi^{(q)}\right)^{-1}\right) \cdot$ $\mathcal{F}) \circ \Phi^{(q)}$. To describe easily an step of the iterative process we write $\mathcal{F}, \mathcal{Z}, \mathcal{R}, \tilde{\mathcal{Z}}, \tilde{\mathcal{R}}$ and $\psi$ instead of $\mathcal{F}^{(q)}, \mathcal{Z}^{(q)}, \mathcal{R}^{(q)}, \mathcal{Z}^{(q+1)}, \mathcal{R}^{(q+1)}$ and $\psi^{(q+1)}$. Now, following the Lie series method for vector fields, we can consider $\psi$ as the flow at unit time of an autonomous system $\dot{X}=\mathcal{S}(X)$ verifying $\mathcal{S}=\square \mathcal{S}$, that we know is a reversible transformation. This leads to $\psi^{*} \mathcal{F}=\Omega+\mathcal{Z}+\mathcal{R}+[\Omega, \mathcal{S}]+r_{2}(\Omega, \mathcal{S})+r_{1}(\mathcal{Z}+\mathcal{R}, \mathcal{S})$, where we have defined $r_{m}(\mathcal{G}, \mathcal{T})=\sum_{k \geq m} \frac{1}{k !} L_{\mathcal{T}}^{k} \mathcal{G}$, with $L_{\mathcal{T}} \mathcal{G}=[\mathcal{G}, \mathcal{T}]=(D \mathcal{T}) \mathcal{G}-(D \mathcal{G}) \mathcal{T}$ being the Lie bracket. Thus we seek for $\Delta \dot{\mathcal{Z}} \in \boldsymbol{R}(\mathcal{M}, K)$ and $\mathcal{S}$ solving the equation

$$
[\mathcal{S}, \Omega]+\Delta \mathcal{Z}=\mathcal{R}_{\leq K}
$$

where $\mathcal{R}_{\leq K}=\sum_{|k|_{1} \leq K} \mathcal{R}_{k}(I) e^{i k \cdot \varphi}$. The solution of this equation in terms of Fourier coefficients is standard. So, the new vector field can be written as $\tilde{\mathcal{F}}=\Omega+\tilde{\mathcal{Z}}+\tilde{\mathcal{R}}$, with

$$
\begin{aligned}
& \tilde{\mathcal{Z}}=\mathcal{Z}+\Delta \mathcal{Z} \in \boldsymbol{R}(\mathcal{M}, K), \\
& \tilde{\mathcal{R}}=\mathcal{R}>K+r_{2}(\Omega, \mathcal{S})+r_{1}(\mathcal{Z}+\mathcal{R}, \mathcal{S}),
\end{aligned}
$$

where we write $\mathcal{R}_{>K}=\mathcal{R}-\mathcal{R}_{\leq K}$.

\section{Norms for functions and vector fields}

In order to obtain estimates for the successive remainders we need to define norms for the vector fields and functions used during the iterative process.

We consider $(I, \varphi)$ with $I \in B_{0} \subset \mathbb{R}^{n}, \varphi \in \mathbb{T}^{n}$. Then, given $\mathcal{U}:=(u, 1) \in \mathbb{R}^{2 n}$ where $u=(u, u, \ldots, u), u>0,1=(1,1, \ldots, 1)$ and $\eta=\left(\eta_{I}, \eta_{\varphi}\right) \in\left(\mathbb{R}_{+} \backslash\{0\}\right)^{2 n}$ we define a complex neighborhood of $B_{0} \times \mathbb{T}^{n}$ as $D_{\eta}=D_{\eta}\left(B_{0}\right)=B_{\eta_{I}} \times V_{\eta_{\varphi}}^{n}$, where $\eta=\tilde{\eta} \mathcal{U}$, being $\tilde{\eta}>0, \tilde{\eta} \in \mathbb{R}$, and

$$
\begin{aligned}
B_{\eta_{I}} & =\bigcup_{I^{*} \in B_{0}}\left\{I \in \mathbb{C}:\left|I_{j}-I_{j}^{*}\right|<\eta_{I_{j}}, j=1,2, \ldots, n\right\}, \\
V_{\eta_{\varphi}}^{n} & =\left\{\varphi: \Re \varphi \in \mathbb{T}^{n},\left|\Im \varphi_{j}\right|<\eta_{\varphi_{j}}, j=1,2, \ldots, n\right\},
\end{aligned}
$$

where $|\cdot|$ denotes the Euclidean norm. For $g: \mathbb{C}^{n} \longrightarrow \mathbb{C}$, we define $|g|_{\eta_{I}}:=\sup _{I \in B_{\eta_{I}}}|g(I)|$ and for an analytic function $g: \mathbb{C}^{n} \times \mathbb{T}^{n} \longrightarrow \mathbb{C}$, and $\eta=\left(\eta_{I}, \eta_{\varphi}\right) \in\left(\mathbb{R}_{+} \backslash\{0\}\right)^{2 n}$ we define

$$
\|g\|_{2, \eta}:=\left(\left|g_{0}\right|_{\eta_{I}}^{2}+\sum_{k \in \mathbb{Z}^{n} \backslash\{0\}}\left|g_{k}(I)\right|_{\eta_{I}}^{2} e^{2|k|_{1} \eta_{\varphi}}\right)^{1 / 2} .
$$


In this way, if $\mathcal{F}: D_{\eta}=B_{\eta_{I}} \times V_{\eta_{\varphi}}^{n} \longrightarrow \mathbb{C}^{2 n}$ is an analytic vector field, $\mathcal{F}=(F, f)$, we write $\|\mathcal{F}\|_{\eta}:=\|\mathcal{F}\|_{2, \eta}:=\max \left\{\|F\|_{2, \eta},\|f\|_{2, \eta}\right\}$, where $\|F\|_{2, \eta}:=\frac{1}{u} \max _{1 \leq j \leq n}\left\|F_{j}\right\|_{2, \eta}$ and $\|f\|_{2, \eta}:=\max _{1 \leq j \leq n}\left\|f_{j}\right\|_{2, \eta}$. Also, if $\mathcal{F}: D_{\eta} \longrightarrow \mathbb{C}^{2 n}$ we consider the norm $\|\mathcal{F}\|_{\infty, 2, \eta}:=$ $\|\mathcal{F}\|_{\infty, \eta}:=\sup _{(I, \varphi) \in D_{\eta}}|\mathcal{F}(I, \varphi)|$.

Remark 1 From now on we will write $\|\cdot\|_{\eta}$ and $\|\cdot\| \|_{\eta}$ to express $\|\cdot\|_{2, \eta}$ and $\|\cdot\| \|_{2, \eta}$, respectively.

Then, one has the following estimates:

Lemma 1 Let $\mathcal{F}=(F, f)$ an analytic vector field on $D_{\eta}=B_{\eta_{I}} \times V_{\eta_{\varphi}}^{n}$ and $\sigma$ such that $0<\sigma<\eta, \sigma=\tilde{\sigma} \mathcal{U}=\tilde{\sigma}(u, 1), u \geq 1$. Assume that $\|\mathcal{F}\|_{\eta}<\tilde{\sigma}$. Then:

a) The flow at unit time associated to $\mathcal{F}, \psi_{1}^{\mathcal{F}}: D_{\eta-\sigma} \longrightarrow D_{\eta}$, is an analytic diffeomorphism and verifies $\left\|\psi_{1}^{\mathcal{F}}-i d\right\|_{\infty, \eta} \leq \alpha_{2}\|\mathcal{F}\|_{\eta}$, with $\alpha_{2}=\alpha_{2}(n)$. Also, if $\mathcal{G}$ is a vector field, analytic on $D_{\eta}$, then $\left(\psi_{1}^{\mathcal{F}}\right)^{*} \mathcal{G}$ is analytic on $D_{\eta-\sigma}$ and it can be represented by its Lie series.

b) Assume that $\|\mathcal{F}\|_{\eta}<\frac{\tilde{\sigma}}{8 \alpha_{1}}$. Then, for any integer $m \geq 0$ the following bound holds

$$
\left\|r_{m}(\mathcal{G}, \mathcal{F})\right\|\left\|_{\eta-\sigma} \leq \alpha_{3}\right\| \mathcal{F}\left\|_{\eta}^{m}\right\| \mathcal{G} \|_{\eta},
$$

where $\alpha_{3}=\alpha_{3}\left(m, \tilde{\sigma}, \alpha_{1}\right)=\beta(m)\left(\frac{4 e \alpha_{1}}{\tilde{\sigma}}\right)^{m}$ and $\beta(m)=\sum_{l=m}^{\infty} \frac{(l-m) !}{l !}$.

\section{The iterative process}

In this section we obtain estimates for one step of the procedure presented in section 2 . Given a module $\mathcal{M}$, an integer $K$ and $\alpha>0$, we say that a subset $L \subset \mathbb{R}^{n}$ is $\alpha, K$ nonresonant modulo $\mathcal{M}$ if

$$
|k \cdot v| \geq \alpha \quad \forall k \in \mathbb{Z}^{n} \backslash \mathcal{M}, \quad|k|_{1} \leq K, \quad \forall v \in L .
$$

Proposition 1 Let $\Omega(I), \mathcal{Z}(I, \varphi)$ and $\mathcal{R}(I, \varphi)$ be vector fields real analytic on $D_{\eta}=$ $D_{\eta}(G)$, where $\Omega(I)=(0, \omega(I))$ and $\mathcal{Z} \in \boldsymbol{R}(\mathcal{M}, K)$. Assume that $\omega(G)$ is $\alpha, K$-nonresonant modulo $\mathcal{M}$ and that $\left|\frac{\partial \omega}{\partial I}\right|_{\eta_{I}} \leq M, \eta_{I} \leq \frac{\alpha}{2 M K}$. Then, the vector fields $\Delta \mathcal{Z}$ and $\mathcal{S}$ which solve the equation (1) are both real analytic on $D_{\eta}$, and the following bounds hold

$$
\|\mathcal{S}\|_{\eta} \leq \frac{2 A}{\alpha}\|\mathcal{R}\|_{\eta}, \quad\|\Delta \mathcal{Z}\|_{\eta} \leq\|\mathcal{R}\|_{\eta}, \quad\|\mathcal{R}-\Delta \mathcal{Z}\|_{\eta} \leq\|\mathcal{R}\|_{\eta},
$$

where

$$
A:=1+\frac{2 \alpha_{2} M}{\alpha}
$$

Theorem 1 (Iterative Lemma) Let $\mathcal{F}(I, \varphi)=\Omega(I)+\mathcal{Z}(I, \varphi)+\mathcal{R}(I, \varphi)$ real analytic reversible on $D_{\eta}$, where $\Omega(I)=(0, \omega(I))$ and $\mathcal{Z} \in \boldsymbol{R}(\mathcal{M}, K)$. Assume that $\omega(G)$ is $\alpha, K$ nonresonant modulo $\mathcal{M}$ and $\left|\frac{\partial \omega}{\partial I}\right|_{\eta_{I}} \leq M$. Let $0<\sigma<\eta$ such that $\sigma=\left(\sigma_{I}, \sigma_{\varphi}\right)=\tilde{\sigma} \mathcal{U}=$ $\tilde{\sigma}(u, 1), u \geq 1$ and let $A$ defined as in (2). Assume

$$
\eta_{I} \leq \frac{\alpha}{2 M K}, \quad\|\mathcal{R}\|_{\eta}<\frac{\alpha_{2} \alpha \sigma_{I}}{16 A} .
$$


Then, there exists a real analytic reversible transformation $\psi_{1}^{\mathcal{S}}: D_{\eta-\frac{\sigma}{2}}(G) \longrightarrow D_{\eta}(G)$ such that $\psi_{1}^{\mathcal{S}^{*}} \mathcal{F}=\Omega+\tilde{\mathcal{Z}}+\tilde{\mathcal{R}}$ with $\tilde{\mathcal{Z}} \in \boldsymbol{R}(\mathcal{M}, K)$, and one has the following bounds

a) $\|\tilde{\mathcal{Z}}\|_{\eta} \leq\|\mathcal{Z}\|_{\eta}+\|\mathcal{R}\|_{\eta}$,

b) $\|\tilde{\mathcal{R}}\|_{\eta-\sigma} \leq e^{-K \sigma_{\varphi}}\|\mathcal{R}\|_{\eta}+\frac{12 A \alpha_{2}^{3}}{\tilde{\sigma} \alpha}\left(\|\mathcal{Z}\|_{\eta}+\|\mathcal{R}\|_{\eta}\right)\|\mathcal{R}\|_{\eta}$,

c) $\left\|\psi_{1}^{\mathcal{S}}-i d\right\|_{\infty, \eta-\frac{\sigma}{2}} \leq \frac{2 A \alpha_{2}^{2}}{\alpha}\|\mathcal{R}\|_{\eta}$,

d) $\psi_{1}^{\mathcal{S}}\left(D_{\eta^{\prime}}(G)\right) \supset D_{\eta^{\prime}-\frac{\sigma}{2}}(G)$ for $\eta^{\prime} \leq \eta-\frac{\sigma}{2}$.

\section{Stability estimates}

\subsection{ESTIMATES FOR THE NORMAL FORM}

Theorem 2 (Normal Form Theorem) Let $\mathcal{F}(I, \varphi)=\Omega(I)+\mathcal{Z}(I, \varphi)+\mathcal{R}(I, \varphi)$ real analytic reversible on $D_{\eta}$, where $\Omega(I)=(0, \omega(I))$ and $\mathcal{Z} \in \boldsymbol{R}(\mathcal{M}, K)$. Assume that $\omega(G)$ is $\alpha, K$-nonresonant modulo $\mathcal{M}$ and $\left|\frac{\partial \omega}{\partial I}\right|_{\eta_{I}} \leq M$. Let $0<\sigma<\eta$ such that $\sigma=\left(\sigma_{I}, \sigma_{\varphi}\right)=$ $\tilde{\sigma} \mathcal{U}=\tilde{\sigma}(u, 1), u \geq 1$ and let $A$ defined as in (2). Assume

$$
\eta_{I} \leq \frac{\alpha}{2 M K}, \quad\|\mathcal{Z}\|_{\eta}+\|\mathcal{R}\|_{\eta}<\frac{\alpha \sigma_{I}}{70 A K \alpha_{2}^{3} \sigma_{\varphi} u} .
$$

Then, there exists a real analytic reversible transformation $\Phi: D_{\eta-\sigma}(G) \longrightarrow D_{\eta}(G)$ such that $\Phi^{*} \mathcal{F}=\Omega+\mathcal{Z}^{*}+\mathcal{R}^{*}$ with $\mathcal{Z}^{*} \in \boldsymbol{R}(\mathcal{M}, K)$, and that verifies

a) $\left\|\mathcal{Z}^{*}\right\|_{\eta} \leq\|\| \mathcal{Z}\left\|_{\eta}+2\right\|\|\mathcal{R}\|_{\eta}$,

b) $\left\|\mathcal{R}^{*}\right\|_{\eta-\sigma} \leq 3 e^{-\frac{K \sigma_{\varphi}}{2}}\|\mathcal{R}\|_{\eta}$,

c) $\|\Phi-i d\|_{\infty, \eta-\sigma} \leq \frac{4 A \alpha_{2}^{2}}{\alpha}\|\mathcal{R}\|_{\eta}$,

d) $\Phi\left(D_{\eta^{\prime}}(G)\right) \supset D_{\eta^{\prime}-\frac{\sigma}{2}}(G)$ for $\eta^{\prime} \leq \eta-\sigma$.

Remark 2 This result is essentially equivalent to the analogous one in [3] for the Hamiltonian case.

\subsection{NON-RESONANT STABILITY ESTIMATES}

We will apply these results to the case of a perturbation of $n$ harmonic oscillators with frequencies satisfying Diophantine conditions. First we present the estimates in the nonresonant general case.

Lemma 2 Let $\mathcal{F}(I, \varphi)=\Omega(I)+\mathcal{Z}(I, \varphi)+\mathcal{R}(I, \varphi)$ real analytic reversible on $D_{\eta}=D_{\eta}(G)$, where $\Omega(I)=(0, \omega(I))$ and $\mathcal{Z} \in \boldsymbol{R}(\mathcal{M}, K)$. Assume that $\omega(G)$ is $\alpha, K$-nonresonant modulo 0 and $\left|\frac{\partial \omega}{\partial I}\right|_{\eta_{I}} \leq M$. Assume also

$$
\eta_{I} \leq \frac{\alpha}{2 M K \alpha_{2}}, \quad\|\mathcal{Z}\|_{\eta}+\|\mathcal{R}\|_{\eta}<\frac{\alpha \eta_{I}}{140 K \alpha_{2}^{3} \eta_{\varphi} u}
$$

Then, for every trajectory $(I(t), \varphi(t))$ of $\mathcal{F}$, with $(I(0), \varphi(0)) \in G \times \mathbb{T}^{n}$, one has

$$
|I(t)-I(0)| \leq \frac{24 \alpha_{2}^{2}}{\alpha}\|\mathcal{R}\|_{\eta} \quad \text { for } \quad|t| \leq \frac{10}{\alpha} e^{\frac{K \eta_{\varphi}}{6}} .
$$


Theorem 3 Let $\mathcal{F}(I, \varphi)=\Omega(I)+\tilde{\mathcal{Z}}(I, \varphi)$ real analytic reversible on $D_{\eta}=D_{\eta}(G)$, where $\Omega(I)=(0, \omega(I))$. Assume that the frequency vector $\omega$ is $\tau, \gamma$-Diophantine for some $\tau \geq n-1$ and $\gamma>0:|k \cdot \omega| \geq \gamma|k|^{-\tau}, \forall k \in \mathbb{Z}^{n} \backslash\{0\}$. Assume also

$$
\epsilon:=\|\tilde{\mathcal{F}}\|_{\eta} \leq \epsilon_{0}=\frac{\gamma \eta_{I}}{140 \alpha_{2}^{3} \eta_{\varphi} u} .
$$

Then, for every trajectory $(I(t), \varphi(t))$ of $\mathcal{F}$, with $(I(0), \varphi(0)) \in G \times \mathbb{T}^{n}$, one has

$$
|I(t)-I(0)| \leq \frac{\eta_{I}}{11 \eta_{\varphi}}\left(\frac{\epsilon}{\epsilon_{0}}\right)^{\frac{1}{\tau+1}} \quad \text { for } \quad|t| \leq \frac{10}{\gamma} \exp \left\{\frac{\eta_{\varphi}}{12}\left(\frac{\epsilon}{\epsilon_{0}}\right)^{\frac{1}{\tau+1}}\right\} .
$$

\section{Acknowledgements}

The authors are indebted to M. Matveyev for pointing out an important mistake in the first version and for very stimulating discussions. This work has been partially supported by the EC grant ERBCHRXCT-940460. Research by A. Delshams is also supported by the Spanish grant DGICYT PB94-0215 and the Catalan grant CIRIT 1996SGR-00105.

\section{References}

1. V.I. Arnol'd. Nonlinear and Turbulent Processes in Physics, pages 1161-1174. Volume 3 , R.Z. Sagdeev, Harwood. New York, edition, 1984.

2. V.I. Arnol'd and M.B. Sevryuk. Oscillations and bifurcations in reversible systems, chapter 2, pages 31-64. Nonlinear Phenomena in Plasma Physics and Hydrodynamics, R.Z. Sagdeev.Mir, 1986.

3. A. Delshams and P. Gutiérrez. Effective stability and KAM theory. January 1995. Preprint. To appear in J. Differential Equations.

4. F. Fassò. Lie series method for vector fields and hamiltonian perturbation theory. J. Appl. Math. Phys., (41):843-864, 1990.

5. G. Gallavotti. Ergodicity, ensembles, irreversibility in boltzmann and beyond. 1994. Preprint. Archived in mp_arc@math.utexas.edu, \#94-66.

6. G. Gallavotti and E.G.D. Cohen. Dynamical ensembles in stationary states. 1995. Preprint. Archived in mp_arc@math.utexas.edu, \#95-32.

7. N. N. Nekhorosev. An exponential estimate of the time of stability of nearly-integrable Hamiltonian systems. Russian Math. Surveys, 32(6):1-65, 1977.

8. J. Pöschel. Nekhoroshev estimates for quasi-convex Hamiltonian systems. February 1992. 3rd. version.

9. J.A.G. Roberts and G.R.W. Quispel. Chaos and time-reversal symmetry. Order and Chaos in reversible systems. Physics Reports, 2-3(216):63-177, 1992.

10. M.B. Sevryuk. Lower-dimensional tori in reversible systems. Chaos, 1(2):160-167, 1991.

11. M.B. Sevryuk. On invariant tori of reversible systems in the neighbourhood of an equilibrium position. Russian Math. Surveys, 42(4), 1987.

12. M.B. Sevryuk. Reversible systems. Volume 1211 of Lecture Notes in Mathematics, Springer, Berlin, 1986. 